Ecological Applications, 21(7), 2011, pp. 2833-2839

(C) 2011 by the Ecological Society of America

\section{An alternative view of ecological community thresholds and appropriate analyses for their detection: comment}

4 May 2010

To the Editor:

Many studies have shown that physical, chemical, and biological conditions of streams are altered by urban development in their watersheds (see reviews by Walsh et al. 2005b, Wenger et al. 2009). A recent study by Cuffney et al. (2010; hereafter CEA) contributed important new evidence linking urbanization to significant changes in stream communities among nine metropolitan areas (MAs) across the USA. The authors used linear and lowess regression to analyze the responses of macroinvertebrate community metrics to a gradient of urban intensity (Metropolitan Area National Urbanization Intensity Index, or MA-NUII) among 28-30 small watersheds in each MA. One of the stated goals of this study involved analyzing the shape of community response to urbanization in order to "determine if responses are consistent with the expectations of the urban stream syndrome and whether they display the response forms hypothesized by Booth et al. (2004), King et al. (2005), and Walsh et al. (2005a)." CEA contrasted these "actual" empirical response curves with a "theoretical" threshold curve as competing "hypotheses," which they then compared to observations across MAs.

Based on their regression analyses, CEA concluded that "responses of communities showed little evidence to support the existence of an initial response threshold that would have indicated resistance to change at low levels of urbanization" but rather "responses were linear or had a higher rate of change at lower levels of urbanization than at higher levels." CEA synthesized these results (Table 11) and categorized the shape of the response relationships as either "A" ("nearly linear"), "B" ("rapid initial rate of change followed by a decreased rate of change"), and "C" ("initial period of resistance (no change in slope) followed by a rapid change in response"). The authors categorized all but 4 of 54 different metric responses as type "A" or "B," implying that any level of urbanization will lead to immediate, nearly linear degradation of stream communities.
Although the CEA study represents a commendable national sampling effort, we contend that their data do not support some of their conclusions. We perceive limitations, both conceptual and statistical, with their analyses and interpretation of results. However, we view these limitations as symptomatic of broader conceptual differences among scientists and managers about ecological thresholds and appropriate statistical techniques for their analysis. We categorize these limitations as (1) ambiguity about what constitutes a "threshold response," (2) misuse of regression to "test" different hypothesized response shapes and to predict community change without consideration of uncertainty, and (3) use of univariate community metrics as indicators of community responses to anthropogenic environmental gradients. Here, our goal is to illustrate how different conclusions may be reached using an alternative conceptual and analysis framework for assessing community response to novel environmental gradients.

\section{What constitutes a "threshold response"?}

Groffman et al. (2006) defined an ecological threshold as "the point at which there is an abrupt change in an ecosystem quality, property, or phenomenon, or where small changes in an environmental driver produce large responses in the ecosystem." This definition does not necessarily imply a catastrophic, vertical increase or decrease in the response, preceded and followed by zones of minimal change, which is unrealistic for many ecological responses and corresponds more closely to a regime shift or alternative stable state (see reviews by Folke et al. 2004 and Andersen et al. 2009). However, this interpretation is one that we have frequently encountered in discussions about ecological thresholds with other investigators. In the context of ecological communities, we interpret a threshold to mean that the frequency and/or abundance of taxa will increase or decrease sharply at some level of along an environmental gradient, such that an incremental change in a driver such as urban intensity results in a disproportionately large change in community structure relative to elsewhere along the gradient (Baker and King 2010). Our definition does not preclude the possibility that some taxa may decline prior to or following a synchronous decline in multiple taxa (e.g., Fig. 2), but it is grounded in the fact that many interacting species have coevolved under a narrow range of environmental conditions and thus may be influenced in similar ways by novel environments, either physiologically or through disruption of interspecific interactions (Okland et al. 2009).

Further ambiguity linking theoretical community thresholds and empirical observations is apparent in Fig. 3 of CEA, where the authors conflate notions of 
ecological resistance with the absence of disturbance and utilize their empirical response "hypotheses" inconsistently. In the dose-response paradigm presented in Fig. 3A of CEA, "resistance" assumes exposure occurs all along the gradient. The linear (type A) to sharply linear (type B) responses of community metrics presented in Fig. 3B also require immediate exposure. However, it is important to distinguish alteration of abiotic conditions in the stream from biological responses to that alteration (sensu Lake 2000). Elsewhere, CEA make a conflicting assumption that streams situated in watersheds with low levels of urban intensity are, in fact, not exposed to urban stressors, deeming "physical, chemical, and biological data" at MA-NUII $\leq 10$ as "background conditions" (CEA 2010: Table 14). We suggest that this "resistance" zone at least partially represents a lack of instream exposure. Apart from confounding effects of other land uses (e.g., Booth et al. 2004, King et al. 2005), there is no realistic mechanism for replacement of natural land with a minute parcel of developed land to by itself cause immediate alterations to instream conditions, a relationship that would be required for immediate biological degradation. In the following sections, we also show that immediate biological responses to urbanization are not supported by empirical observations.

\section{Use of linear regression to "test" for threshold responses}

CEA reported numerous least-square regression slopes and intercepts for urbanization responses of three macroinvertebrate community metrics determined to strongly respond in six MAs: community composition, expressed as nonmetric multidimensional scaling scores (MDS1), richness-weighted tolerance values of taxa (RichTol), and richness of Ephemeroptera, Plecoptera, and Trichoptera taxa (EPTr). The authors reported that lowess smoothing was used to determine "patterns of community response" (presented in CAE 2010: Table 11), yet provide insufficient details about how it was used to determine response shapes. Representative scatterplots were not presented to illustrate the response to urban intensity (MA-NUII) nor substantiate fitted relationships to these data (although some scatterplots of the same data are provided in Brown et al. 2009), so readers cannot evaluate concordance between statistical and graphical results, as strongly recommended by Zuur et al. (2010).

We downloaded the data sets used by the authors to examine responses of all three metrics to MA-NUII and impervious cover among each of the six MAs exhibiting the strongest response to urbanization: Atlanta, Georgia; Birmingham, Alabama; Boston, Massachusetts; Portland, Oregon; Raleigh, North Carolina; and Salt Lake City, Utah (data sets available online). ${ }^{1}$ Commu-

\footnotetext{
${ }^{1}\langle$ http://pubs.usgs.gov/ds/423/〉
}

nity metrics used in the CEA analysis were based on two macroinvertebrate collection methods, a quantitative richest-targeted habitat $(\mathrm{RTH})$ and qualitative multihabitat (QMH) approach (Cuffney et al. 2010). We also downloaded a third set of metrics computed by USGS but not used by CEA based on a combination of the RTH and QMH data (QQ). The QQ metrics should represent the most precise estimate of taxa richness and composition given greater coverage of habitat types, increased sampling effort, and given that richness metrics were the "most useful in assessing community response".

Preliminary graphical analysis suggested that metric responses to MA-NUII and impervious cover were approximately linear for much of the gradient, but metrics did not respond immediately and linearly at the lowest levels of urbanization as reported by CEA. Graphical analysis (Zuur et al. 2010) of residuals from least-squares linear regressions revealed that they were consistently falling below or above the regression line at low levels of urbanization. Following the authors' use of lowess regression to determine response shapes, we fit local polynomial regressions to the metric responses to MA-NUII using the loess function in $\mathrm{R} 2.9 .2$ ( $\mathrm{R}$ Development Core Team 2009) and used the default span value of 0.75 (a smoothing parameter). We computed $95 \%$ confidence limits along the fitted regression line to assess uncertainty in estimates of MA-NUII that produced a significant change in each community metric.

Fig. 1 illustrates the response of EPTr (computed from RTH samples) for each of the six MAs reported as having strong responses to urbanization. In all six cases, the loess regression line is flat at low levels of MA-NUII and does not significantly decline (according to 95\% CIs) until MA-NUII reaches 25 (Atlanta), 25 (Birmingham), 13 (Boston), 10 (Portland), 27 (Raleigh), and 20 (Salt Lake City). A simple scatterplot of the relationship between metrics and MA-NUII values between 0 and 10 (Boston, Portland) and 0 and 20-25 (Atlanta, Birmingham, Raleigh, Salt Lake City) is sufficient to reveal a pattern closely resembling a flat line with a slope at or near zero (a pattern evident in the vast majority of the other metric responses to MA-NUII per our reanalysis of CEA data sets; Appendix ). However, relatively few sample units limit strong inference. In particular, the Salt Lake City results are not statistically defensible because virtually no data exists between 0 and 20 MANUII (Fig. 1; Appendix). Regardless of collection method (RTH, QMH, QQ), our analysis of response patterns in EPTr and other metrics (MDS1, RichTol) were most similar to the threshold response type $\mathrm{C}$, rather than $\mathrm{A}$ and $\mathrm{B}$, as reported by the authors.

Despite small sample sizes and nonlinear responses, CEA extended their approach to predict percent change in community metrics relative to "background condi- 

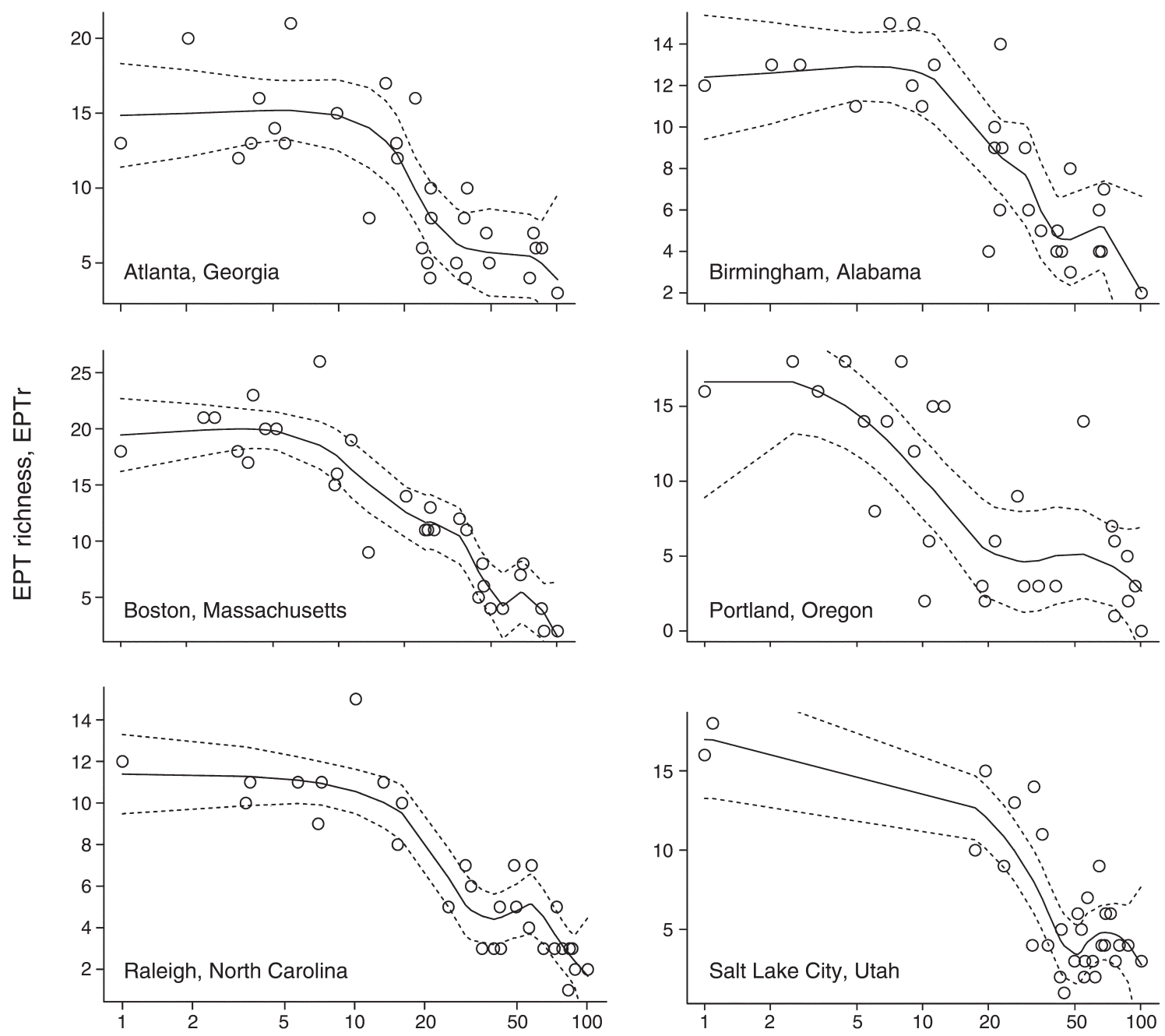

Metropolitan Area National Urbanization Intensity Index (MA-NUII)

FIG. 1. Loess regressions of Ephemeroptera-Plecoptera-Trichoptera (EPT) richness on the Metro Area National Urbanization Intensity Index (MA-NUII) for six U.S. metropolitan areas studied by Cuffney et al. (2011). The $x$-axis is displayed on a log scale to more effectively illustrate the lack of change at low levels of urban intensity. However, regressions were conducted using untransformed values of MA-NUII.

tions" (in this case, "intercepts") for $5 \%$ and $10 \%$ impervious cover (as estimated by MA-NUII). These predictions (CEA: Table 15) were presented as part of their discussion without estimation of error between predictions at either $5 \%$ or $10 \%$ impervious cover and the intercept ( $0 \%$ impervious cover). Thus, the estimates of percent assemblage change at $5 \%$ and $10 \%$ impervious cover are directly analogous to comparing the difference between two means without considering variance. Yet even if the comparisons included estimates of uncertainty, they would still be based on a poorly justified linear model (Fig. 1, Appendix). Finally, Table 15 in CEA reports identical predicted values of MA-NUII and percent change in macroinvertebrate assemblages at 5\% and $10 \%$ impervious cover. The perfect correspondence between these results appears to be in error.

\section{Aggregate community metrics obscure taxon-specific responses}

Perhaps our most critical concern with the analytical approach offered by CEA and other similar studies (e.g., Booth et al. 2004, Moore and Palmer 2005, Walsh et al. 2005a, Paul et al. 2009; see reviews by Brenden et al. 2008, Andersen et al. 2009, Dodds et al. 2010) stems from their use of aggregate response metrics to detect and interpret the magnitude of community change. Embedded in the interpretation of such metrics are a host of implicit assumptions about the nature and 


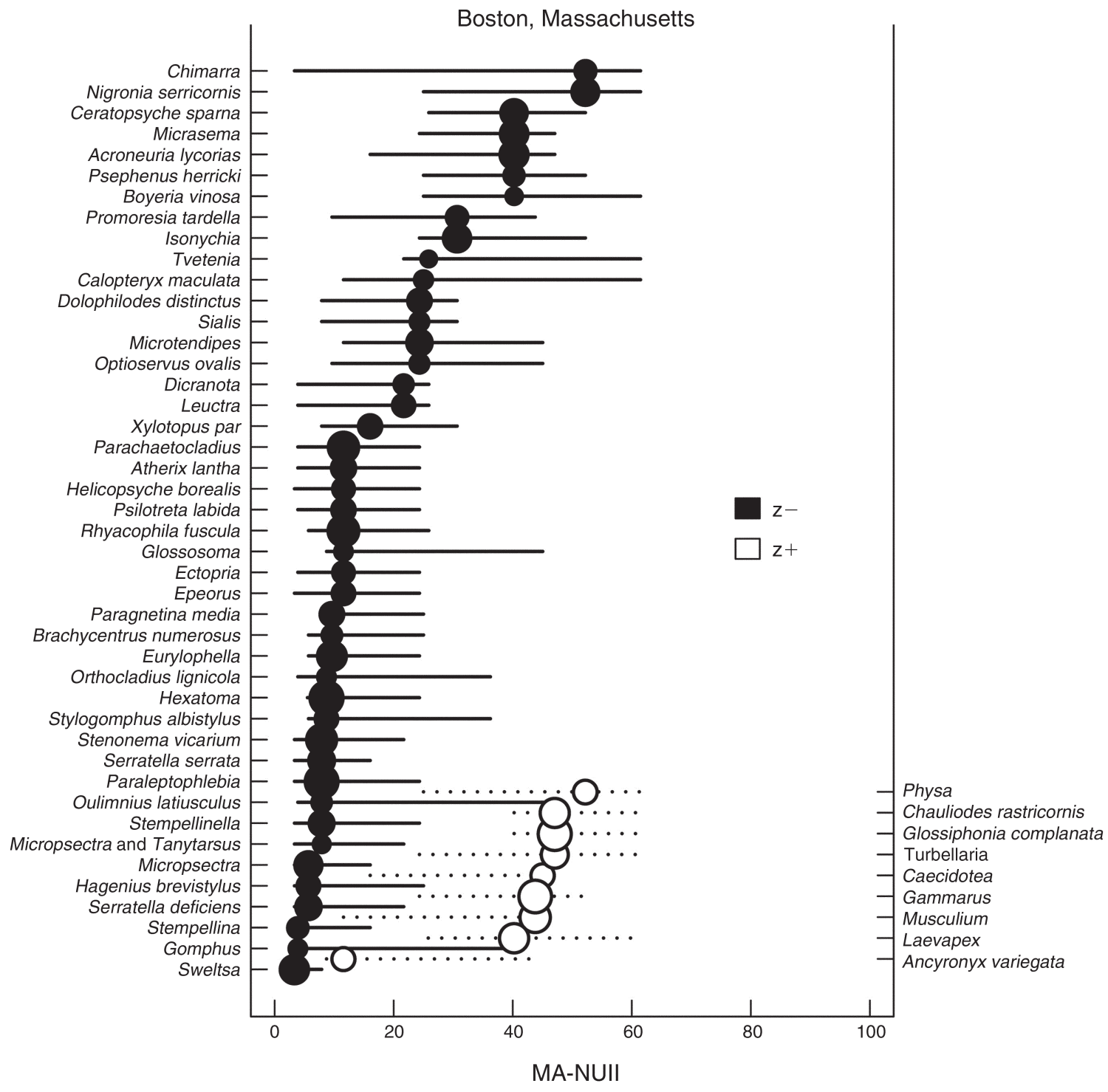

FIG. 2. Plot of taxon-specific change-point maxima along a gradient of urbanization intensity for two metropolitan areas studied by Cuffney et al. (2011). Declining taxa $(\mathrm{z}-)$ are listed on the left axis and represented by solid symbols proportional to their response magnitude. Increasing taxa $(\mathrm{z}+)$ are similarly listed on the right axis and represented by open symbols. Horizontal lines show $95 \%$ quantiles around change points as computed from 500 bootstrap replicates. Only taxa with reliably significant and directionally pure responses during resampling are shown.

direction of change. For example, a priori designation of groups of taxa for use in richness metrics combines losses of certain taxa with potential gains of others as well as noise contributed by unresponsive taxa (King and Baker 2010). Because change points along the gradient can vary dramatically across related taxa, because the magnitude of these changes differ, because the direction of the response can differ, and because many taxa add noise through lack of response, we view reliance on community metrics to be a fundamental flaw in any "test" for community thresholds. Moreover, interpretation of metric responses assumes that differ- ences between sites represent the response shape of community "change" despite confounding potential nonlinear declines with more gradual increases in weedy taxa (Baker and King 2010).

We recently developed threshold indicator taxa analysis (TITAN) to address the challenges described above and to supplement aggregate metrics and univariate methods for threshold identification (Baker and King 2010). TITAN finds the value of an environmental gradient that produces the greatest change in species abundance and occurrence within a sample population. Association with one side of an 


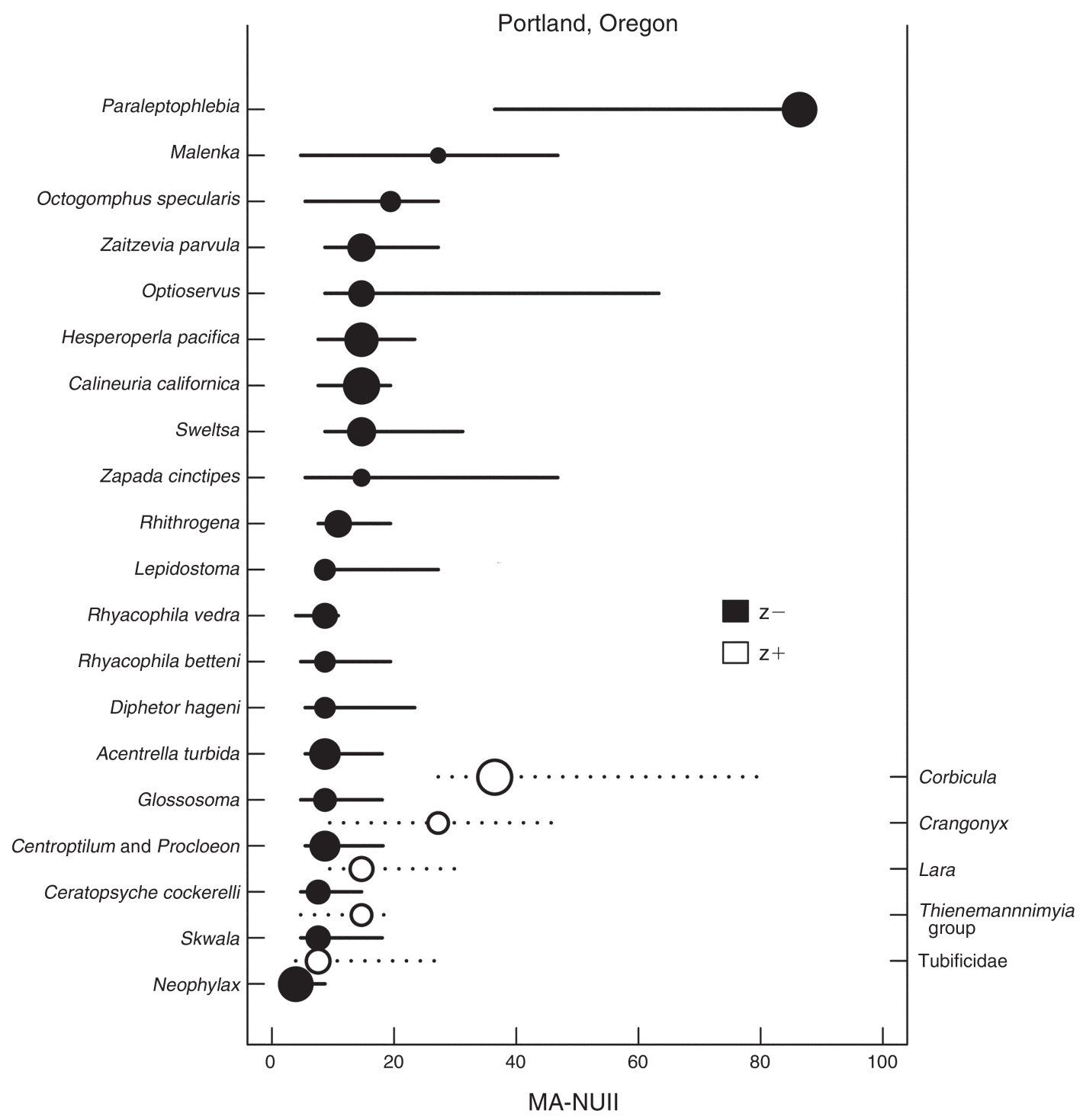

FIG. 2. Continued.

environmental change point is measured by indicator species analysis values (Dufrêne and Legendre 1997), and normalized (as $z$ scores) by comparison with values obtained from permuted samples. TITAN distinguishes between increasing and declining taxa while facilitating comparison of response magnitude. Community response is tracked by summing the normalized change among increasing or decreasing taxa separately, and evidence of a community threshold is assessed by synchronous change among taxa. Uncertainty in taxon-specific or community change points along the environmental gradient is assessed by a bootstrap procedure. To more fully understand community response in the CEA data sets, we performed TITAN in R.2.9.2 (Baker and King 2010).
Fig. 2 illustrates the distribution of taxa change points in two representative MAs with strong "linear" response types according to CEA. Both panels showed sharp, nearly synchronous change points for the majority of negative-responding indicator taxa between 5 and 15 MA-NUII. Boston showed additional taxa declines at higher levels of urbanization consistent with a threshold definition inclusive of additional change beyond a point of disproportionate change. In contrast, increasers were far fewer and had change points anywhere between 10 and 60 MA-NUII. However, locations of increasing taxa change points were less synchronous and more uncertain than many decliners, a pattern that we have documented along urbanization gradients in the Baltimore-Washington MA (Baker and King 2010, King and 
TABLE 1. Results from threshold indicator taxa analysis (TITAN) using two sets of USGS National Water Quality Assessment Program (NAWQA) macroinvertebrate abundance data (richest targeted habitat [RTH] and Qualitative Multihabitat [QQ]) in response to the Metropolitan Normalized Urban Intensity Index (MA-NUII) and impervious cover (\%) in six metropolitan areas of the USA.

\begin{tabular}{|c|c|c|c|c|c|c|}
\hline \multirow[b]{2}{*}{ Metropolitan area } & \multicolumn{3}{|c|}{ RTH } & \multicolumn{3}{|c|}{ QQ } \\
\hline & $\mathrm{CP}$ & $5 \%$ & $95 \%$ & $\mathrm{CP}$ & $5 \%$ & $95 \%$ \\
\hline \multicolumn{7}{|l|}{ MA-NUII } \\
\hline Atlanta & 22.3 & 6.1 & 25.5 & 22.3 & 6.9 & 25.5 \\
\hline Birmingham & 29.1 & 5.0 & 29.1 & 25.4 & 5.0 & 29.1 \\
\hline Boston & 11.6 & 3.9 & 25.0 & 11.6 & 3.9 & 25.9 \\
\hline Portland & 8.7 & 5.4 & 19.4 & 7.6 & 5.4 & 23.4 \\
\hline Raleigh & 26.7 & 6.1 & 40.0 & 26.7 & 13.2 & 36.9 \\
\hline Salt Lake City & 32.9 & 20.5 & 51.6 & 32.9 & 24.0 & 43.0 \\
\hline \multicolumn{7}{|c|}{ Impervious cover $(\%)$} \\
\hline Atlanta & 6.1 & 1.3 & 6.6 & 2.6 & 1.3 & 6.8 \\
\hline Birmingham & 6.3 & 1.4 & 11.3 & 7.9 & 1.8 & 10.3 \\
\hline Boston & 3.7 & 1.0 & 7.4 & 3.7 & 1.0 & 8.5 \\
\hline Portland & 2.4 & 1.0 & 9.2 & 3.3 & 1.3 & 8.4 \\
\hline Raleigh & 5.3 & 2.6 & 12.1 & 5.3 & 2.6 & 12.1 \\
\hline Salt Lake City & 12.5 & 7.0 & 22.7 & 10.4 & 10.4 & 22.7 \\
\hline
\end{tabular}

Notes: Change points (CP) and confidence limits $(5 \%, 95 \%)$ correspond to the values of the predictor resulting in the largest aggregate change in negative-responding taxa $[\operatorname{sum}(z-)]$ in the observed data and 500 bootstraps, respectively. Metropolitan areas are Atlanta, Georgia; Birmingham, Alabama; Boston, Massachusetts; Portland, Oregon; Raleigh, North Carolina; and Salt Lake City, Utah.

Baker 2010). In both cases, observed changes in community structure across the gradient would be poorly characterized as "linear."

Fundamentally, the majority of the taxa in both data sets were too variable in their responses to statistically categorize as negative or positive, yet many (EPTr) or all (RichTol, MDS1) unresponsive taxa are included in the univariate aggregations used by CEA. This may be the most important limitation of aggregate community metrics in threshold analyses. We have shown using real and simulated community data (King and Baker 2010) that the aggregation of the sharp, synchronous declining taxa with more asynchronous increasing taxa or unresponsive taxa into community metrics can explain the linear or wedge-shaped response prevalent across studies linking urbanization to stream community response (e.g., Booth et al. 2004, Moore and Palmer 2005, Walsh et al. 2005b, Paul et al. 2009). Disaggregation of such data sets with TITAN demonstrates synchronous changes in many taxa and strong evidence for community thresholds obscured by the aggregate measures employed by CEA.

Further analysis of all six MAs using TITAN also revealed synchronous declines in multiple taxa, which were manifested as distinct local maxima (sharp peaks) in the sum of individual indicator taxa responses [sum $(z-)$ ] indicative of a community threshold (Table 1; see Baker and King 2010 for further explanation). As in the loess fits, small sample sizes per MA led to some uncertainty about change point location, but observed MA-NUII and impervious cover change points were remarkably consistent among MAs. We have demonstrated similarly low, but much more precise thresholds in studies of nearly 300 watersheds along urbanization gradients in Maryland (0.8-3.4\% watershed development [Baker and King 2010]; 0.5-2\% impervious cover [King and Baker 2010]).

Collectively, the CEA data, our previous analyses, and other work (e.g., Utz et al. 2009) support the conclusion that alarmingly low levels of urbanization lead to rapid changes in biological condition of streams. However, the linear response model proposed by CEA neither adequately nor accurately conveys the location or magnitude of biodiversity losses at low levels of urbanization. We remain concerned about the ambiguity of theoretical response models based on use of aggregate community metrics and the corresponding use of linear, univariate statistical approaches for their analysis because of their potential to mislead scientists and policymakers. We recommend an integration of a more precise definition of thresholds, ecologically grounded conceptual models, graphical analysis of data prior to statistical modeling, awareness of the potential for long gradients to graphically obscure low-level thresholds, and analytical approaches that disaggregate communities to consider taxon-specific responses in future assessment of urbanization and other anthropogenic gradients.

\section{Acknowledgments}

The authors contributed equally to this paper.

\section{Literature cited}

Andersen, T., J. Carstensen, E. Hernandez-Garcia, and C. M. Duarte. 2009. Ecological regime shifts: approaches to identification. Trends in Ecology and Evolution 24:49-57. 
Baker, M. E., and R. S. King. 2010. A new method for detecting and interpreting biodiversity and ecological community thresholds. Methods in Ecology and Evolution 1: 25-37.

Booth, D. B., J. R. Karr, S. Schauman, C. P. Konrad, S. A. Morley, M. G. Larson, and S. J. Burges. 2004. Reviving urban streams: land use, hydrology, biology, and human behavior. Journal of the American Water Resources Association 40:1351-1364.

Brenden, T. O., L. Wang, and Z. Su. 2008. Quantitative identification of disturbance thresholds in support of aquatic resource management. Environmental Management 42:821832.

Brown, L. R., T. F. Cuffney, J. F. Coles, F. Fitzpatrick, G. McMahon, J. Steuer, A. H. Bell, and J. T. May. 2009. Urban streams across the USA: lessons learned from studies in 9 metropolitan areas. Journal of the North American Benthological Society 28:1051-1069.

Cuffney, T. F., R. B. Brightbill, J. T. May, and I. R. Waite. 2010. Responses of benthic macroinvertebrates to environmental changes associated with urbanization in nine metropolitan areas. Ecological Applications 20:1384-1401.

Dodds, W. K., W. H. Clements, K. Gido, R. H. Hilderbrand, and R. S. King. 2010. Thresholds, breakpoints, and nonlinearity in freshwaters as related to management. Journal of the North American Benthological Society 29:988-997.

Dufrêne, M., and P. Legendre. 1997. Species assemblages and indicator species: the need for a flexible asymmetrical approach. Ecological Monographs 67:345-366.

Folke, C., S. Carpenter, B. Walker, M. Scheffer, T. Elmqvist, L. Gunderson, and C. S. Holling. 2004. Regime shifts, resilience, and biodiversity in ecosystem management. Annual Review of Ecology, Evolution, and Systematics 35:557-581.

Groffman, P. M., et al. 2006. Ecological thresholds: They key to successful environmental management or an important concept with no practical application? Ecosystems 9:1-13.

King, R. S., and M. E. Baker. 2010. Considerations for detecting and interpreting ecological community thresholds in response to anthropogenic environmental gradients. Journal of the North American Benthological Society 29:998-1008.

King, R. S., M. E. Baker, D. F. Whigham, D. E. Weller, P. F. Kazyak, and M. K. Hurd. 2005. Spatial considerations for linking watershed land cover to ecological indicators in streams. Ecological Applications 15:137-153.

Lake, P. S. 2000. Disturbance, patchiness, and diversity in streams. Journal of the North American Benthological Society 19:473-592.

Moore, A. A., and M. A. Palmer. 2005. Invertebrate biodiversity in agricultural and urban headwater streams: implications for conservation and management. Ecological Applications 15:1169-1177.

Okland, B., O. Skarpaas, and K. Kausrud. 2009. Threshold facilitations of interacting species. Population Ecology 51:513-523.

Paul, M. J., D. W. Bressler, A. H. Purcell, M. T. Barbour, E. T. Rankin, and V. H. Resh. 2009. Assessment tools for urban catchments: defining observable biological potential. Journal of the American Water Resources Association 45:320-330.

R Development Core Team. 2009. R 2.9.2 for Windows. R Project for Statistical Computing, Vienna, Austria. 〈www. r-project.org $\rangle$

Utz, R. M., R. H. Hildebrand, and D. M. Boward. 2009. Indentifying regional differences in threshold responses of aquatic invertebrates to land cover gradients. Ecological Indicators 9:556-567.

Walsh, C. J., T. D. Fletcher, and A. R. Ladson. 2005a. Stream restoration in urban catchments through redesigning stormwater systems: looking to the catchment to save the stream. Journal of the North American Benthological Society 24:690-705.

Walsh, C. J., A. H. Roy, J. W. Feminella, P. D. Cottingham, P. M. Groffman, and R. P. Morgan. 2005b. The urban stream syndrome: current knowledge and the search for a cure. Journal of the North American Benthological Society 24:706-723.

Wenger, S. J., et al. 2009. Twenty-six key research questions in urban stream ecology: an assessment of the state of the science. Journal of the North American Benthological Society 28:1080-1098.

Zuur, A. F., E. N. Ieno, and C. S. Elphick. 2010. A protocol for data exploration to avoid common statistical problems. Methods in Ecology and Evolution 1:3-14.

\section{RYAN S. KING}

Center for Reservoir and Aquatic Systems Research Department of Biology

Baylor University

One Bear Place \#97388

Waco, Texas 76798 USA

E-mail: Ryan_S_King@baylor.edu

Matthew E. Baker

Department of Geography and Environmental Systems

University of Maryland-Baltimore County

Baltimore, Maryland 21205 USA

\section{APPENDIX}

Scatterplots of community metrics in response to low levels of urban intensity (MA-NUII) among six U.S. metropolitan areas studied by Cuffney et al. (2010) (Ecological Archives A021-127-A1). 